\title{
Anti-neutrophil cytoplasmic antibodies in cholesterol embolism: A case report and literature review
}

\author{
JUN ZHANG, HENG-YUAN ZHANG, SHI-ZHI CHEN and JI-YI HUANG \\ Department of Nephrology, The First Affiliated Hospital of Xiamen University, Xiamen, Fujian 361003, P.R. China
}

Received March 18, 2015; Accepted April 22, 2016

DOI: $10.3892 /$ etm.2016.3349

\begin{abstract}
Cholesterol embolism is a multisystemic disorder with clinical manifestations that resemble vasculitis. Anti-neutrophil cytoplasmic antibodies (ANCA) are a defining feature of ANCA-associated vasculitis, and the presence of ANCA in cholesterol embolism complicates its differential diagnosis and treatment. At present, the role of ANCA in cholesterol embolism remains unclear and no effective treatment is currently available. The present study reports the case of an Asian male who presented with spontaneous cholesterol embolism with proteinase 3 (PR3)-specific ANCA, subacute interstitial nephritis and late-developing skin lesions. The 69-year-old patient was admitted to The First Affiliated Hospital of Xiamen University (Xiamen, China) complaining of chest tightness, fatigue, progressive renal failure and refractory hypertension. In addition, transient eosinophilia was detected. Following immunosuppressive therapy with steroids and cyclophosphamide for 6 months, hemodialysis treatment was initiated. Skin lesions appeared at $>1$ month following hemodialysis initiation; however, they were gradually improved following treatment with atorvastatin and anti-platelet aggregation therapy for 5 months. The patient was maintained on hemodialysis for $\sim 2$ years and exhibited general good health at the most recent follow-up. In addition, 11 cases of cholesterol embolism associated with ANCA reported in the literature were discussed in the present study.
\end{abstract}

\section{Introduction}

Cholesterol embolism, which is also known as cholesterol emboli syndrome, cholesterol crystal embolism or atheroembolism, is a relatively rate but potentially serious complication of atherosclerosis (1). Cholesterol emboli occur spontaneously or iatrogenically following vascular surgery, catheterization

Correspondence to: Dr Ji-Yi Huang, Department of Nephrology, The First Affiliated Hospital of Xiamen University, 55 Zhenhai Road, Xiamen, Fujian 361003, P.R. China

E-mail: heymann@163.com

Key words: cholesterol embolism, antineutrophil cytoplasmic antibodies, renal disease, steroids, cyclophosphamide or anticoagulation treatment when cholesterol crystals located in an atherosclerotic plaque in a large caliber artery embolize to small or medium caliber arteries, resulting in obstructive end-organ damage and an inflammatory response (2). Previous studies have reported that the incidence of cholesterol embolism is $0.31-2.4 \%$ (3). In recent years, the incidence of cholesterol embolism has increased due to a greater frequency of triggering factors, typically catheterization or vascular surgery. Elderly individuals with diabetes, hypertension, and dyslipidaemia are particularly susceptible to cholesterol embolism (3). As there is no definitive treatment for cholesterol embolism, therapeutic strategies are predominantly preventive and supportive (3). Prognosis is poor; $33 \%$ of patients who require renal replacement therapy succumb to their symptoms during the first year (4).

Cholesterol embolism is ubiquitous; therefore, the clinical manifestation are protean, non-specific, insidious and multi-systemic (3). Diagnosis is challenging, and can only be achieved via a biopsy. Vasculitis is an important differential diagnosis (5). Cholesterol embolism and anti-neutrophil cytoplasmic antibodies (ANCA)-associated vasculitis (AAV) are distinct entities; however, their clinical manifestations, including renal failure, skin lesions and elevation of inflammatory markers, are markedly similar (6). ANCA are useful indices for the diagnosis of AAV (7). Therefore, a ANCA-positive result in patients with cholesterol embolism may complicate its differential diagnosis and treatment.

In order to deepen the understanding of differential diagnosis and treatment between cholesterol embolism and AAV, the present study reports a case of cholesterol embolism in an Asian male, who presented with subacute progressive nonoliguric renal failure, refractory hypertension, late-developing skin lesions and proteinase 3 targeting ANCA (PR3-ANCA).

\section{Case report}

Prior to admission to The First Affiliated Hospital of Xiamen University (Xiamen, China), the 69-year-old male described in the present case report had been admitted to a regional hospital in May 2010 complaining of chest tightness, shortness of breath and fatigue. The patient had been treated with statins, calcium channel blockers, an angiotensin receptor antagonist, a diuretic, antibiotics and acetylsalicylic acid. Upon demonstrating a normal renal function, with serum creatinine $(\mathrm{Scr})$ levels of $1.13 \mathrm{mg} / \mathrm{dl}$ [normal range $(\mathrm{NR}), 0.49-1.50 \mathrm{mg} / \mathrm{dl}$ ] 
A

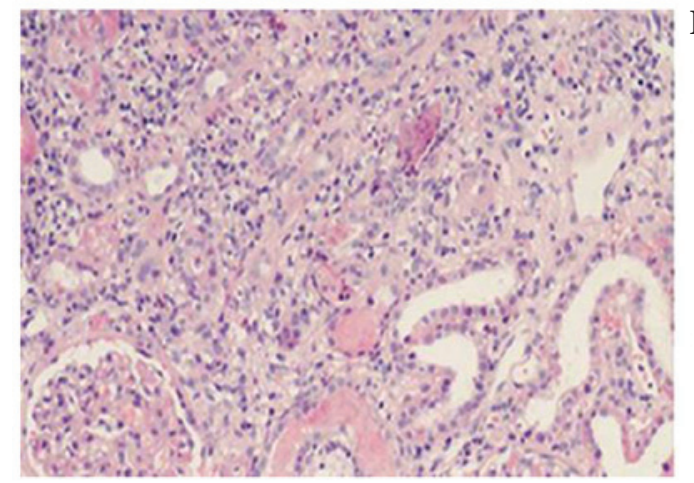

B

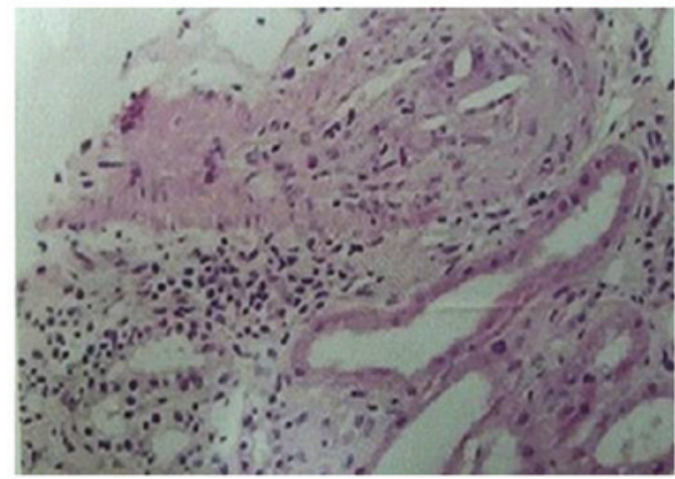

Figure 1. Renal biopsy of the patient, as detected by hematoxylin and eosin staining. (A) Renal interstitium was saturated with inflammatory cells, including eosinophils, neutrophils, monocytes, lymphocytes and plasmocytes, as detected by H\&E staining (magnification, x100). (B) Needle shaped cholesterol emboli were detected in the lumen of the interlobular arteries (magnification, $x 400$ ).

and normal urinalysis results, the patient was discharged. However, during a 2-week follow-up period, a gradual rise in Scr levels $(2.45 \mathrm{mg} / \mathrm{dl})$ was detected, as well as leukocytosis and eosinophilia. Leukocytosis and eosinophilia peaked in July 2010, and the white blood cell (WBC) count was $16.6 \times 10^{9} / 1$ (NR, 4-10.0 $\left.\times 10^{9} / 1\right)$, with $15.8 \%$ eosinophils $\left(2.62 \times 10^{9} / 1\right.$; $\left.\mathrm{NR}, 0.02-0.5 \times 10^{9} / 1\right)$. Urine volume and urinalysis results remained normal. Atherosclerotic plaques were detected in the femoral and popliteal arteries of the bilateral lower limbs by a color Doppler ultrasound; however, the reason for transient eosinophilia and renal failure was not elucidated. Scr levels fluctuated around $1.83 \mathrm{mg} / \mathrm{dl}$ (NR, 0.49-1.50 mg/dl) for several months.

The patient was admitted to the Department of Cardiology at The First Affiliated Hospital of Xiamen University in January 2011. The patient had a 30-year history of smoking and suffered from chronic pulmonary disease, gout, hypertension, hypercholesterolemia, coronary artery disease and diabetes mellitus. A physical examination and subsequent blood tests demonstrated the following: Blood pressure, $183 / 98 \mathrm{mmHg}$ (NR, <140/90 mmHg); WBC, $10.1 \times 10^{9} / 1$ without eosinophils; hemoglobin, $8.8 \mathrm{~g} / \mathrm{dl}$ (NR, 11-16 g/dl); erythrocyte sedimentation rate (ESR), $82 \mathrm{~mm} / \mathrm{h}(\mathrm{NR}, 0-15 \mathrm{~mm} / \mathrm{h})$; blood urea nitrogen, $50.1 \mathrm{mg} / \mathrm{dl}$ (NR, 8.12-22.96 mg/dl); creatinine, $3.4 \mathrm{mg} / \mathrm{dl}(\mathrm{NR}, 0.49-1.50 \mathrm{mg} / \mathrm{dl})$; total cholesterol, $272.2 \mathrm{mg} / \mathrm{dl}$ (NR, 108.3-216.6 mg/dl); C-reactive protein (CRP), $5.4 \mathrm{mg} / \mathrm{dl}$ (NR, 0-3 mg/dl); 24-h proteinuria, $7.64 \mathrm{~g}$ (NR, $<0.15 \mathrm{~g}$ ); urine sediments containing mild microhematuria; a normal platelet count; and no cutaneous lesions. Tests for anti-double stranded DNA antibodies, anti-phospholipid antibody, rheumatoid factor, hepatitis $\mathrm{B}$ virus, hepatitis $\mathrm{C}$ virus and human immunodeficiency virus were negative, and serum complement levels were normal. Tests for myeloperoxidase ANCA (MPO-ANCA) and anti-glomerular basement membrane antibodies were negative, whereas the results of a PR3-ANCA test were positive (50.6 RU/ml; NR, <20 RU/ml). Bilateral bronchial pneumonia and left pulmonary lingular hamartoma were detected and nasal endoscopy demonstrated rhinitis, under armor hypertrophy and erosion in the left plow zone.

In order to control the patient's blood pressure, combined antihypertensive drugs (5 mg amlodipine besylate b.i.d., $80 \mathrm{mg}$ valsartan q.d., $25 \mathrm{mg}$ hydrochlorothiazid q.d., $20 \mathrm{mg}$ furosemide

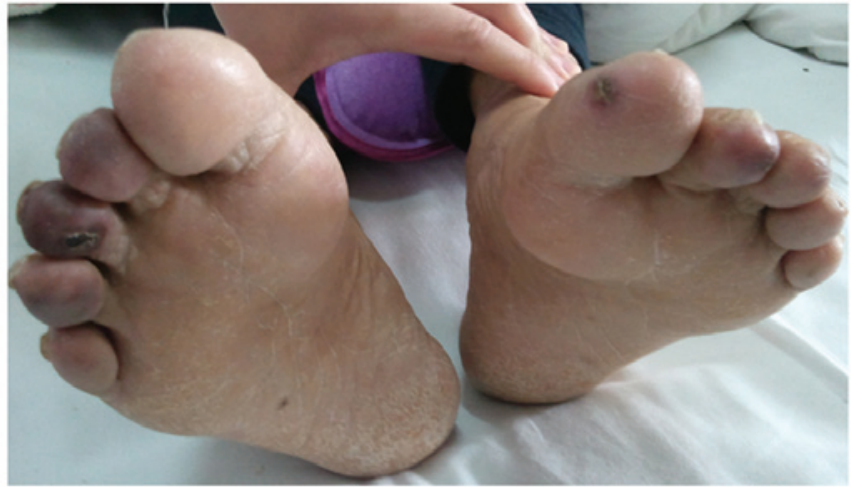

Figure 2. Necrosis or gangrene of the patient's toes following hemodialysis.

q.d., $1 \mathrm{mg}$ prazosin t.i.d., and intravenous sodium nitroprusside or nitroglycerin) were administered; however, the patient's blood pressure fluctuated (180-200/93-107 $\mathrm{mmHg}$ ) and his renal function deteriorated (Scr, $5.42 \mathrm{mg} / \mathrm{dl})$. As a result of a further decline in renal function and refractory hypertension, the patient was transferred to the Department of Nephrology at The First Affiliated Hospital of Xiamen University, where he was treated with statins (20 mg atorvastatin calcium tablets p.o. q.n.; Pfizer Inc., New York, NY, USA), an antibiotic (4.5 g piperacillin/tazobactam i.v. g.t.t. q12 h; Pfizer Inc.) and an anti-aggregant (75 mg clopidogrel hydrogen sulfate p.o. q.d.; Sanofi S.A., Paris, France), and his renal function subsequently improved (Scr decreased to $3.5 \mathrm{mg} / \mathrm{dl}$ ).

In March 2011, the patient underwent a renal biopsy. Renal biopsy specimens were examined by light and immunofluorescence microscopy. For light microscopy, specimens were fixed in $10 \%$ buffered-formaldehyde, embedded in paraffin, and $2 \mu \mathrm{m}$ sections were stained with hematoxylin and eosin, periodic acid-Schiff and periodic acid-silver methenamine. For immunofluorescence examination, the specimens were embedded in an optimal cutting temperature compound (Miles Laboratories, Elkhart, IN, USA) and frozen in an acetone-dry ice mixture. Frozen sections were cut into 5- $\mu \mathrm{m}$ on a cryostat, rinsed in $0.01 \mathrm{~mol} / 1$ phosphate-buffered saline (PBS; $\mathrm{pH} 7.4$ ), fixed in absolute acetone for $10 \mathrm{~min}$, and incubated for $30 \mathrm{~min}$ at room temperature with fluorescein isothiocyanate-conjugated rabbit antihuman $\operatorname{IgG}, \operatorname{IgA}, \operatorname{IgM}, \mathrm{Clq}, \mathrm{C} 3, \mathrm{C} 4$ or fibrinogen 


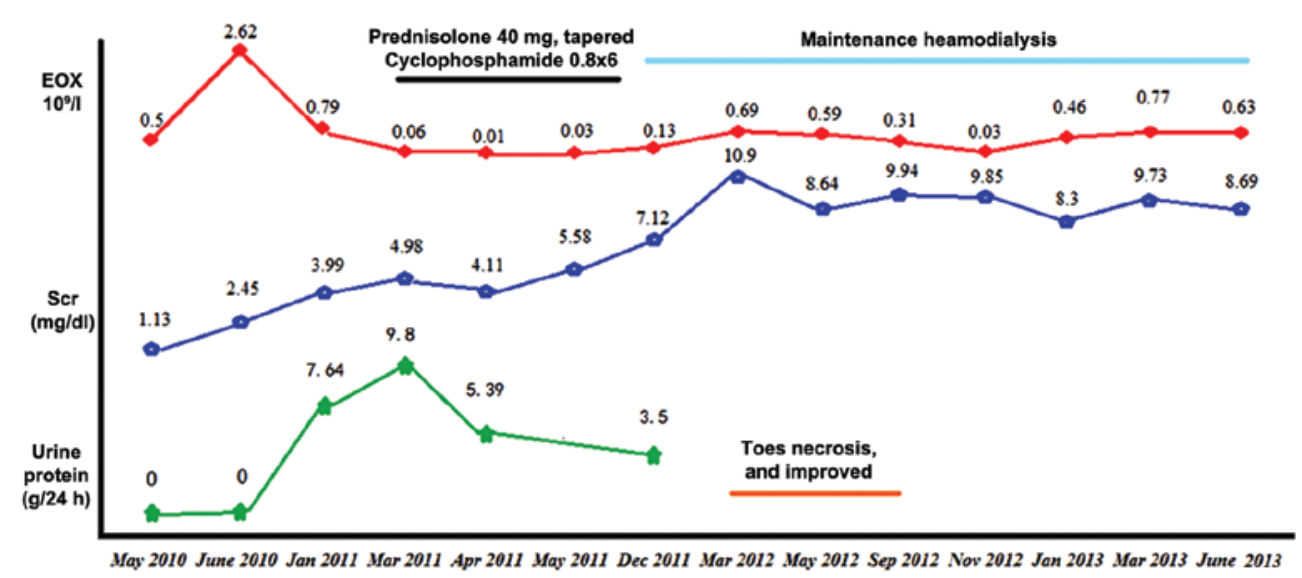

Figure 3. Clinical course of the present case. EO, eosinophilia; Scr, serum creatinine.

antisera (Dakopatts, Copenhagen, Denmark). Stained sections were rinsed in PBS and examined under a fluorescence photomicroscope (Zeiss Axiophot, Oberkochen, Germany). Analysis of the specimen demonstrated 40 glomeruli in the renal cortex, of which 16 exhibited global sclerosis and two exhibited segmental sclerosis. The remaining glomeruli were diminished to varying extents. Extracapillary proliferation, crescents or segmental necrotizing lesions were not detected in the glomeruli. Tubular atrophy was severe and the renal interstitium exhibited mild edema and fibroplastic proliferation. In addition, the renal interstitium was saturated with inflammatory cells, including eosinophils, neutrophils, monocytes, lymphocytes and plasmocytes (Fig. 1A). As shown in Fig. 1B, the vascular wall of the interlobular arteries was thickened, and the lumen of the interlobular arteries was narrowed. Furthermore, individual interlobular arteries exhibited hyalinosis, and needle-shaped cholesterol emboli were observed in the respective lumens of two interlobular arteries (Fig. 1B). However, neither immunoglobulin nor complement deposition were observed, according to a routine immunofluorescence analysis.

Taking all the results together, the patient was diagnosed with cholesterol embolism and subacute interstitial nephritis, associated with PR3-ANCA. Immunosuppressive therapy with a steroid, prednisone, and cyclophosphamide (CTX; both SINE Shanghai Pharma Co., Ltd., Shanghai, China) was initiated. A total of $40 \mathrm{mg}$ prednisone was initially administered per day; however, the dosage was reduced to $30 \mathrm{mg} /$ day after 4 weeks, and was subsequently tapered gradually at 2- or 4-week intervals until suspension at 6 months. CTX (0.8 g) was administered intravenously six times at 4-week intervals. During the course of immunosuppressive therapy, Scr levels fluctuated (4.43-5.96 mg/dl), and urinalysis (Aution Max AX-4030, Arkray, Tokyo, Japan) revealed nephritic proteinuria and trace hematuria. PR3-ANCA titer decreased to $35.3 \mathrm{RU} / \mathrm{ml}$; however, it remained positive at the end of immunosuppressive therapy.

In December 2011, due to a further decline in renal function (Scr level, $7.52 \mathrm{mg} / \mathrm{dl}$ ), hemodialysis was performed three times per week. A month later, cyanosis was observed on the patient's toes and he complained of severe pain. In addition, the right pedal pulse was weakened. Color Doppler ultrasound analysis demonstrated atherosclerosis and plaques in the femoral and popliteal arteries of the bilateral lower limbs, and hypoperfusion in the right anteriortibial artery and right dorsalis pedis. Cyanosis of the patient's toes worsened and four toes exhibited signs of necrosis or gangrene (Fig. 2). The patient refused to undergo a skin biopsy of the affected toes and an angiography of the lower limb arteries. Following 5 months of treatment with atorvastatin $(20 \mathrm{mg}$ q.d.; Pfizer, Inc.) and anti-aggregants, including aspirin (100 mg q.d.; Bayer, Shanghai, China), sarpogrelate (100 mg b.i.d.; Mitsubishi Gas Chemical Company, Inc., Tokyo, Japan), clopidogrel (50 mg q.d.; CardinalHealth China, Shanghai, China) and cilostazol (50 mg b.i.d.; Otsuka Pharmaceutical Co., Ltd., Tokyo, Japan), the necrosis gradually improved. The patient was maintained on hemodialysis for $\sim 2$ years (Fig. 3). At the most recent follow-up, the patient exhibited general good health and has been undergoing maintenance hemodialysis in our hemodialysis center for $>4$ years.

\section{Discussion}

Diagnosis of cholesterol embolism remains challenging due to its diverse manifestations. Cholesterol embolism is a well-documented cause of renal disease (8). Renal failure and cutaneous lesions are two of the most common clinical manifestations of cholesterol embolism (3); however, they are also frequently observed in patients with systemic vasculitis (6). Furthermore, the presence of ANCA in cholesterol embolism typically complicates its differential diagnosis and treatment, since ANCA are a defining feature of ANCA-associated vasculitis (AAV) (7). Cholesterol embolism remains a rare disease, therefore its incidence is unclear (3). Cholesterol embolism associated with ANCA has rarely been reported. To date, only 11 cases reported in English could be retrieved from the PubMed database (http://www.ncbi.nlm.nih.gov/pubmed) using the term 'cholesterol embolism plus ANCA' (9-18). Clinical characteristics and laboratory findings of these 12 patients (including the present case) are listed in Table I.

The average age of the patients was 69 years (age range, 47-76 years) and $82 \%$ of the patients were aged $>63$ years. Of the 12 patients reported in the literature, 10 were male, two were female, four were Asian and eight were Caucasian. A 


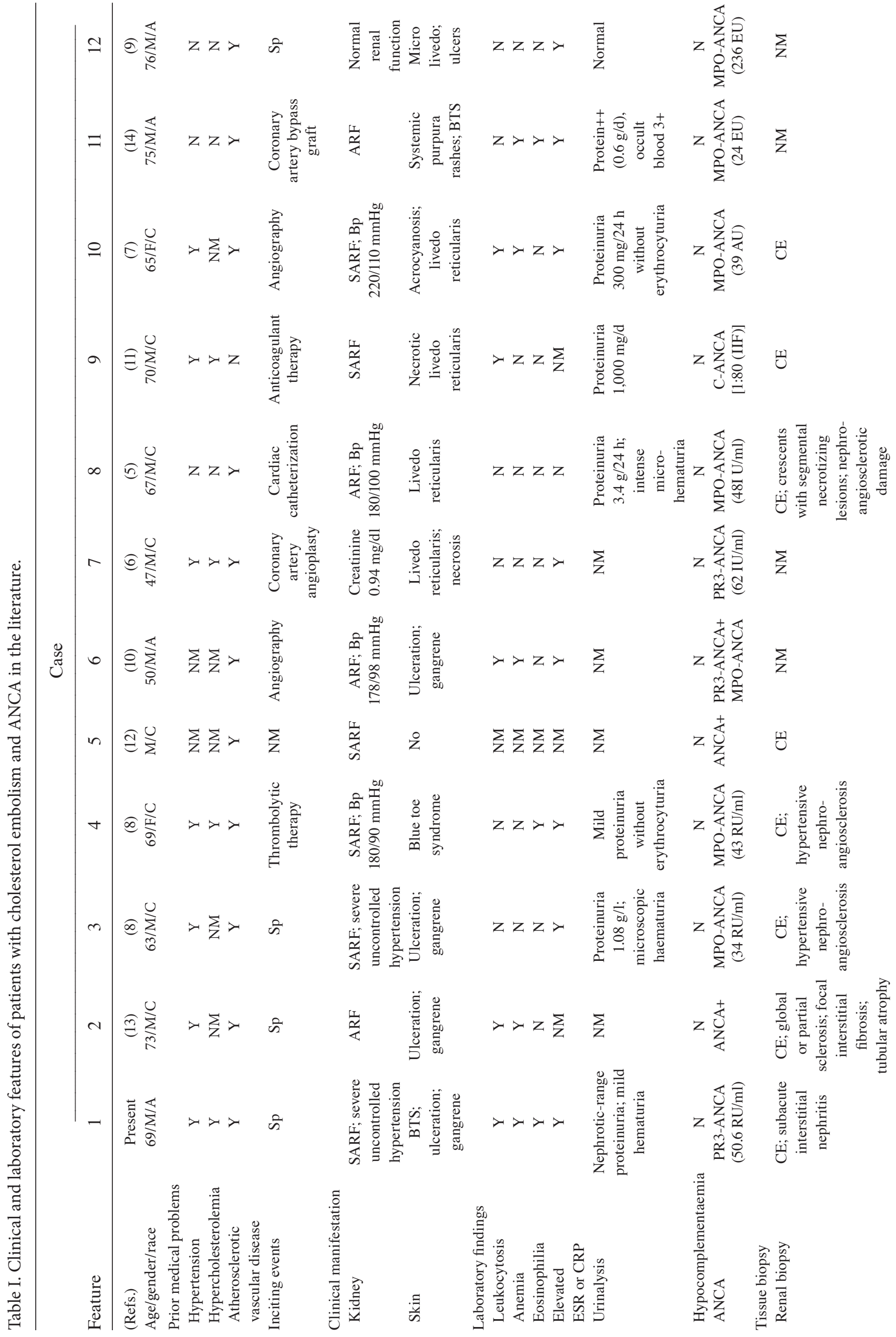


number of these patients suffered from one or more medical problems prior to admission, including hypertension $(n=7)$, hypercholesterolemia $(n=4)$, chronic obstructive pulmonary disease $(n=3)$ and atherosclerotic vascular disease $(n=11)$. In addition, $91 \%$ of the patients suffered from one or more atherosclerotic vascular diseases, including coronary heart disease, abdominal aortic aneurysm, peripheral vascular disease or ischemic nephropathy, and a third of the patients were smokers.

According to the preliminary clinical data, $33 \%$ of the patients (4/12) developed cholesterol embolism spontaneously; whereas the remaining eight patients developed the disease following a vascular procedure, including anticoagulation or thrombolysis therapy (19). Four patients presented with acute renal failure, and pronounced renal impairment typically occurred within 2 weeks. Six patients exhibited subacute renal failure several weeks after an inciting event and two patients demonstrated normal renal function. Notably, 50\% of the patients exhibited severe uncontrolled hypertension. In the present case, the blood pressure of the patient remained difficult to control, despite treatment with antihypertensive drugs. Skin lesions are the most common extrarenal manifestation of cholesterol embolism and the majority of patients suffer from blue toe syndrome, livedo reticularis, ulceration or gangrene (3). Typically, the skin lesions developed at the onset of the disease; however, in the present case, skin lesions were observed at 1.5 years after disease presentation. Since the skin lesions presented within the period of maintained hemodialysis, the episode may have occurred due to the use of anticoagulants for hemodialysis (20).

It has previously been reported that eosinophilia occurs in $80 \%$ of cases of atheroembolic renal disease, therefore it may help to establish the diagnosis (3). However, eosinophilia was only detected in $25 \%$ of the patients (3/12) with cholesterol embolism in the literature. A possible explanation for this discrepancy is that eosinophilia frequently occurs during the acute phase of the disease and is typically transient (3). In the present case, eosinophil counts peaked in the early stage and subsequently fluctuated during the course of the disease. In the majority of patients, elevated levels of inflammatory markers, including ESR and CRP, were also observed. Among the 12 patients, urinalysis results were typically benign with mild hematuria, and only minor proteinuria was detected in the present case. However, there was no record of urinalysis having taken place for four patients. One patient exhibited normal urinalysis results, five patients showed moderate proteinuria with or without hematuria and proteinuria was demonstrated in one patient $(3.4 \mathrm{~g} / 24 \mathrm{~h})$ with intense microhematuria and hyaline granular casts. Nephrotic-range proteinuria, which was observed in the present case, has previously been described in a number of cholesterol embolism patients with focal glomerulosclerosis or diabetic nephropathy $(3,5)$.

Of the 12 patients reported in the literature, three were positive for cytoplasmic ANCA (C-ANCA), six were positive for perinuclear ANCA (P-ANCA), one patient was positive for both C-ANCA and P-ANCA and two patients were ANCA-positive, as determined by early indirect immunofluorescence. ANCA positivity in patients with cholesterol embolism typically confounds the differential diagnosis and treatment of the disease, and the role of ANCA in cholesterol embolism remains unclear. Whether the occurrence of ANCA 
in cholesterol embolism is merely a coincidence or bears cause and effect is yet to be elucidated. In addition, it is unclear whether ANCA underlies the thromboembolic pathogenic process. It has previously been suggested that ANCA may have a pathogenetic role in AAV (21); however, other studies have argued against the involvement of ANCA due to the presence of naturally occurring ANCA in healthy individuals and the lack of an association between ANCA titres and disease activity (7). Furthermore, it has been hypothesized that not all PR3- or MPO-ANCAs are pathogenic (7). A previous study reported that ANCA did not have a major role in premature atherosclerosis, since no increased level of the autoantibody was observed (22). In some cases, including the present case, the presence of ANCA may occur synchronously with cholesterol embolism, resulting in impairment of renal function and systemic inflammation $(11,12,14,15,17)$. In two cases, persistent ANCA and vasculitis developed following cholesterol embolism $(10,16)$. Conversely, in other cases, vasculitis and cholesterol embolism were deemed coexistent, based on the identification of prior medical problems, intense hematuria, variable ANCA titres or a high ANCA titre $(9,13,18)$. Notably, the clinical features of cholesterol embolism with ANCA typically resemble those of patients with cholesterol embolism alone $(3,6)$. These data indicate that cholesterol embolism may enhance the induction of ANCA during neutrophil activation as a result of damage to the vascular system, which is consistent with the induction of ANCA following in silica exposure to or the administration of propylthiouracil (23). However, further large population-based studies are required in order to clarify the role of ANCA in cholesterol embolism.

Histological confirmation is regarded as the definitive method for the diagnosis of cholesterol embolism, and a renal biopsy was performed for eight of the 12 patients with cholesterol embolism in the literature $(9,11,12,15-17)$. Consistent with previous reports $(9,12,17)$, cholesterol emboli were observed in the lumen of the interlobular arteries in the present case, and histological changes to the glomeruli and interstitium were observed. Skin or muscle biopsies were performed for eight of the 12 patients $(11-15,17,18)$. Two cases showed no evidence of vasculitis or cholesterol crystal clefts $(11,15)$, whereas six cases demonstrated cholesterol embolism, of which three also exhibited inflammatory infiltration of neutrophils into the walls of the small arteries, thus indicating that vasculitis and cholesterol embolism may have been coexistent $(12,13,18)$.

The British Society for Rheumatology/British Health Professionals in Rheumatology guideline recommends that newly diagnosed AAV should be assessed for treatment with glucocorticoids and CTX (24). However, at present, no definitive treatment has been established and no clinical trials have been conducted in patients with atheroembolic renal disease; therefore, the majority of therapeutic measures are preventive (3). Although anticoagulants may trigger atheroembolization, the results of a previous study did not support the hypothesis that peritoneal dialysis is superior to hemodialysis (20), and the use of steroids remains controversial (3). Of the cases of cholesterol embolism with positive ANCA, ten (83\%) were treated with steroids, of which six (60\%) were also administered CTX. Combination therapy with steroids and CTX has been shown to be effective in five cases $(9,10,14,16,17)$. One patient improved following treatment with prednisolone and CTX, but relapsed after the prednisolone dosage was reduce and CTX was discontinued (17). Treatment with a steroid alone was effective in three cases, including one case of pleuritis and two cases of cholesterol embolism with vasculitis and cutaneous lesions; the renal functions of these three patients were improved following treatment $(11,13,18)$. One patient was able to discontinue hemodialysis after steroid therapy (18). One patient was treated with hemodialysis and supportive therapy; however, the skin lesions deteriorated and the patient succumbed to multiorgan failure after 12 weeks (15). In the present case, steroids combined with CTX therapy were administered at 8 months post-disease onset, upon a renal biopsy and detection of subacute interstitial nephritis and gradual deterioration of renal function.

In conclusion, the present study reported the case of a 69-year-old Asian male who successively presented with eosinophilia, subacute progressive renal failure, refractory hypertension, PR3-ANCA positivity, cholesterol embolism with interstitial nephritis and late-developing skin lesions. The present study provides an overall perspective on the ANCA-associated cholesterol embolism through a comparative discussion of the clinical data of 11 cases of ANCA-associated cholesterol embolism in the literature and the present case. The roles of ANCA in cholesterol embolism, and efficient treatment strategies for patients with ANCA-associated cholesterol embolism, remain to be elucidated and require further investigation.

\section{Acknowledgements}

The authors would like to thank the staff of the Hemodialysis Unit at The First Affiliated Hospital of Xiamen University.

\section{References}

1. Quinones A and Saric M: The cholesterol emboli syndrome in atherosclerosis. Curr Atheroscler Rep 15: 315-321, 2013.

2. Saric M and Kronzon I: Aortic atherosclerosis and embolic events. Curr Cardiol Rep 14: 342-349, 2012.

3. Scolari F and Ravani P: Atheroembolic renal disease. Lancet 375: 1650-1660, 2010

4. Thériault J, Agharazzi M, Dumont M, Pichette V, Ouimet D and Leblanc M: Atheroembolic renal failure requiring dialysis: Potential for renal recovery? A review of 43 cases. Nephron Clin Pract 94: c11-c18, 2003.

5. Meyrier A: Cholesterol crystal embolism: Diagnosis and treatment. Kidney Int 69: 1308-1312, 2006.

6. Minota S: ANCA in atheroembolism; just a coincidence or bearing cause and effect? Intern Med 45: 495-496, 2006.

7. Schönermarck U, Csernok E and Gross WL: Pathogenesis of anti-neutrophil cytoplasmic antibody-associated vasculitis: Challenges and solutions 2014. Nephrol Dial Transplant 30 (Suppl 1) i46-i52, 2015.

8. Scolari F, Tardanico R, Zani R, Pola A, Viola BF, Movilli E and Maiorca R: Cholesterol crystal embolism: A recognizable cause of renal disease. Am J Kidney Dis 36: 1089-1109, 2000.

9. Aviles B, Ubeda I, Blanco J and Barrientos A: Pauci-immune extracapillary glomerulonephritis and atheromatous embolization. Am J Kidney Dis 40: 847-851, 2002.

10. de RS, Serratrice J, Granel B, Disdier P, Bartoli JM, Pache X, Astoul P, Garbe L, Branchereau A and Weiller PJ: Periaortitis heralding Wegener's granulomatosis. J Rheumatol 29: 392-394, 2002.

11. Delen S, Boonen A, Landewé R, Kroon AA, van der Linden S and Tervaert JW: An unusual case of ANCA positive disease. Ann Rheum Dis 62: 780-781, 2003.

12. Kaplan-Pavlovcic S, Vizjak A, Vene N and Ferluga D: Antineutrophil cytoplasmic autoantibodies in atheroembolic disease. Nephrol Dial Transplant 13: 985-987, 1998. 
13. Maejima H, Noguchi T and Tanei R: Cholesterol embolism associated with MPO-ANCA. Eur J Dermatol 20: 539-540, 2010.

14. Maeshima E, Yamada Y, Mune M and Yukawa S: A case of cholesterol embolism with ANCA treated with corticosteroid and cyclophosphamide. Ann Rheum Dis 60: 726, 2001.

15. Miguélez A, Barrientos N, López-Rios F, Vanaclocha F and Iglesias L: Necrotic livedo reticularis, multiple cholesterol emboli and ANCA. J Eur Acad Dermatol Venereol 17: 351-352, 2003.

16. Palmgren E, Hartford M and Herlitz H: Cholesterol embolism-a serious systemic disease. Lakartidningen 97: 1263-1266, 2000 (In Swedish).

17. Peat DS and Mathieson PW: Cholesterol emboli may mimic systemic vasculitis. BMJ 313: 546-547, 1996.

18. Sugimoto T, Morita Y, Yokomaku Y, Isshiki K, Kanasaki K, Eguchi Y, Koya D and Kashiwagi A: Systemic cholesterol embolization syndrome associated with myeloperoxidase-anti-neutrophil cytoplasmic antibody. Intern Med 45: 557-561, 2006.

19. Scolari F, Ravani P, Gaggi R, Santostefano M, Rollino C, Stabellini N, Colla L, Viola BF, Maiorca P, Venturelli C, et al: The challenge of diagnosing atheroembolic renal disease: Clinical features and prognostic factors. Circulation 116: 298-304, 2007
20. Ravani P, Gaggi R, Rollino C, Santostefano M, Stabellini N, Colla L, Dallera N, Ravera S, Bove S, Faggiano P and Scolari F: Lack of association between dialysis modality and outcomes in atheroembolic renal disease. Clin J Am Soc Nephrol 5: 454-459, 2010.

21. Kallenberg CG: Pathogenesis and treatment of ANCA-associated vasculitides. Clin Exp Rheumatol 33 (4 Suppl 92): S11-S14, 2015.

22. van Haelst PL, Asselbergs FW, van Doormaal JJ, Veeger NJ, May JF, Holvoet P, Gans RO and Tervaert JW: Antineutrophil cytoplasmatic antibodies in patients with premature atherosclerosis: Prevalence and association with risk factors. J Intern Med 251: 29-34, 2002.

23. de Lind van Wijngaarden RA, van Rijn L, Hagen EC, Watts RA, Gregorini G, Tervaert JW, Mahr AD, Niles JL, de Heer E, Bruijn JA and Bajema IM: Hypotheses on the etiology of antineutrophil cytoplasmic autoantibody associated vasculitis: The cause is hidden, but the result is known. Clin J Am Soc Nephrol 3: 237-252, 2008

24. Ntatsaki E, Carruthers D, Chakravarty K, D'Cruz D, Harper L, Jayne D, Luqmani R, Mills J, Mooney J, Venning M, et al: BSR and BHPR guideline for the management of adults with ANCA-associated vasculitis. Rheumatology (Oxford) 53: 2306-2309, 2014 